Classification

Physics Abstracts

$47.50-05.70$

\title{
Phenomenology of polymer migration
}

\author{
D. Lhuillier \\ Laboratoire de Mécanique Théorique $\left(^{*}\right)$, Université P. et M. Curie, \\ 4, place Jussieu, 75230 Paris Cedex 05, France
}

(Reçu le 20 septembre 1982, accepté le 29 novembre 1982)

\begin{abstract}
Résumé. - Dans un mélange, le flux de diffusion de masse s'exprime habituellement comme le gradient d'une différence de potentiel chimique. L'extension de cette loi de Fick aux solutions de polymères déformables a été critiquée et, à partir de la théorie cinétique, Sekhon, Armstrong et Jones, ont récemment suggéré d'écrire sous forme de la divergence d'un tenseur la partie du flux de masse dépendant de la déformation des polymères. On se propose ici, en considérant un mélange polymère-solvant avec une vitesse distincte pour chacun des composants, de montrer comment la thermodynamique macroscopique permet de cerner les hypothèses qui sont à la base de chacune des deux formes proposées. A partir des expressions explicites que nous obtenons il devrait être facile de les départager expérimentalement car la diffusion induite par la déformation des polymères aussi bien que l'effet Soret présentent des caractères très différents d'une forme à l'autre.
\end{abstract}

\begin{abstract}
The diffusion mass flux in a mixture is usually written as the gradient of a chemical potential difference. When generalized to a solution of deformable polymers, this Fick's law leads to difficulties and, on the basis of a kinetic theory approach, Sekhon, Armstrong and Jones recently suggested that that part of the mass flux which depends on the polymer deformation could be written as the divergence of a second-rank tensor. Our aim here is to consider a mixture of polymers and solvent with two different velocities, and with the help of macroscopic thermodynamics, to determine the assumptions leading to each of the two proposed expressions. We then show how the Soret effect and the deformation-induced diffusion are different for the two fluxes. Thus an experimental determination of the right proposal should, in principle, be easy to perform.
\end{abstract}

1. Introduction. - The anomalous retention of polymer molecules inside a porous medium [1-3] or the strange rheological effects observed in a capillary flow [4-6] led to the conclusion that a polymer solution is likely to exhibit spatial concentration gradients in a non-homogeneous flow. The origin of this migration has been long attributed to the deformation entropy of the molecules [7-8] which results in a force driving the polymers towards the regions of lowest stresses, i.e. the regions where the molecules are less deformed.

On the other hand, experiments on DNA molecules in a circular Couette flow [9] demonstrated a migration of these (rather rigid) molecules towards the rotating inner cylinder, the very place where the stress is highest. A second explanation was then proposed [10-11] which links migration to a purely viscous force, the so-called Faxen' force which is a modified form of Stokes' law in a non-homogeneous velocity field [12].

(*) L.A. $n^{\circ} 229$.
Among polymer physicists, the supporters of the viscous force and those of the entropic force form two orthogonal groups. As if this situation was not controversial enough, Sekhon, Armstrong and Jones [13] recently suggested that the hydrodynamic interactions inside the polymer molecules were responsible for a new force (hereafter called the SAJ force) leading to another possibility for polymer migration.

It is noteworthy that the SAJ and Faxen forces can be derived within the dumbbell model for polymers while the entropic force cannot. This is all the more surprising since the entropic force is nothing but a mere generalization of the famous Fick's law to the case of a solution of deformable particles. This situation is rather puzzling and the purpose of this paper is to reconsider the mechanisms of polymer migration with the help of thermodynamics. We will not deal at length with the Faxen's force since it is rather well known and exists even for a rigid particle. Here we focus upon the forces directly depending on the 
polymer deformation and the results we arrive at with our thermodynamic approach are twofold.

- Firstly we determine the (mutually exclusive) hypotheses leading to the entropic force on the one hand, and to a SAJ-like force on the other hand.

- Secondly we find an explicit expression for these forces in terms of the polymer deformation. For the entropic force this is the expression already found by Tirrell and Malone [14]. For the SAJ force the result is new.

2. The free enthalpy of a dilute polymer solution. The free enthalpy per unit volume of a solution of polymer molecules embedded in a solvent can be written as

$$
G=\rho_{\mathrm{p}} \mu_{\mathrm{p}}^{0}+\rho_{\mathrm{s}} \mu_{\mathrm{s}}^{0}+\Delta G_{\mathrm{m}}+\Delta G_{\mathrm{d}}+\Delta G_{\mathrm{k}}
$$

where $\rho_{\mathrm{p}}$ is the polymer mass per unit volume of the solution, $\mu_{\mathrm{p}}^{0}$ is the chemical potential per unit mass in a pure polymer phase while the index $s$ characterizes the same quantities for the solvent.

$\Delta G_{\mathrm{m}}$ is the free enthalpy of mixing; we have a rather complete knowledge of it [15] but we only need its limiting form for dilute solutions (the case we consider henceforth)

$$
\Delta G_{\mathrm{m}}=k T \frac{\rho_{\mathrm{p}}}{m_{\mathrm{p}}} \log \frac{\rho_{\mathrm{p}}}{\rho_{\mathrm{p}}^{0}}
$$

where $m_{\mathrm{p}}$ is the molecular mass of the polymer and $\rho_{\mathrm{p}}^{0}$ the mass per unit volume of the pure polymer phase. Hence $\rho_{\mathrm{p}} / \rho_{\mathrm{p}}^{0}$ is the volume fraction of the polymers and $\rho_{\mathrm{p}} / m_{\mathrm{p}}$ is the number of polymers per unit volume of the solution.

$\Delta G_{\mathrm{d}}$ is the free enthalpy of deformation. For a polymer in a « bad » solvent, $\Delta G_{\mathrm{d}}$ may be approximated as the deformation entropy of a Gaussian chain [16]. When the deformation is slight, as is the case in Poiseuille or Couette flow, Flory's complete expression merges into the simple result [17]

$$
\Delta G_{\mathrm{d}}=\frac{1}{2} k T \frac{\rho_{\mathrm{p}}}{m_{\mathrm{p}}}\left(C_{i j}-\delta_{i j}\right)^{2}
$$

where

$$
C_{i j}=\frac{3\left\langle r_{i} r_{j}\right\rangle}{r_{0}^{2}}
$$

is the adimensional shape of the polymers and $r_{0}$ their equilibrium radius of gyration. For a polymer in a " good" solvent, the interactions between the monomers can be represented phenomenologically by considering $r_{0}$ as some function of the temperature and in this case $\Delta G_{d}$ can no longer be thought of as a purely entropic contribution.

Lastly $\Delta G_{\mathbf{k}}$ is a kinematic contribution occurring when the velocities $V_{p}$ and $V_{s}$ are different from the centre of mass velocity $\mathbf{V}$ defined as

$$
\rho \mathbf{V}=\rho_{\mathrm{p}} \mathbf{V}_{\mathrm{p}}+\rho_{\mathrm{s}} \mathbf{V}_{\mathrm{s}}
$$

with

$$
\rho=\rho_{\mathrm{p}}+\rho_{\mathrm{s}} \text {. }
$$

If we neglect any " added mass " effect, i.e. the extra kinetic energy obtained by the solvent because of the polymer motion, we can simply write

$$
\begin{aligned}
\Delta G_{\mathrm{k}} & =\frac{1}{2} \rho_{\mathrm{p}}\left(\mathbf{V}_{\mathrm{p}}-\mathbf{V}\right)^{2}+\frac{1}{2} \rho_{\mathrm{s}}\left(\mathbf{V}_{\mathrm{s}}-\mathbf{V}\right)^{2} \\
& =\frac{1}{2} \frac{\rho_{\mathrm{p}} \rho_{\mathrm{s}}}{\rho}\left(\mathbf{V}_{\mathrm{p}}-\mathbf{V}_{\mathrm{s}}\right)^{2} .
\end{aligned}
$$

The differential form of the free enthalpy is then

$$
\begin{aligned}
\mathrm{d} G=\mu_{\mathrm{p}} \mathrm{d} \rho_{\mathrm{p}}+\mu_{\mathrm{s}} \mathrm{d} \rho_{\mathrm{s}}-S \mathrm{~d} T+ & \alpha_{i j} \mathrm{~d} C_{i j}+ \\
& +P_{i} \mathrm{~d} w_{i}+\mathrm{d} p
\end{aligned}
$$

where $p$ is the pressure while for dilute solutions, we have

$$
\begin{gathered}
\mu_{\mathrm{p}}=\frac{\partial G}{\partial \rho_{\mathrm{p}}}=\mu_{\mathrm{p}}^{0}+\frac{k T}{m_{\mathrm{p}}}\left[\log \frac{\rho_{\mathrm{p}}}{\rho_{\mathrm{p}}^{0}}+\right. \\
\left.+\frac{1}{2}\left(C_{i j}-\delta_{i j}\right)^{2}\right]+ \\
\mu_{\mathrm{s}}=\frac{\partial G}{\partial \rho_{\mathrm{s}}}=\mu_{\mathrm{s}}^{0}+\frac{1}{2}\left(\mathbf{V}_{\mathrm{p}}-\mathbf{V}\right)^{2} \\
S=-\frac{\partial G}{\partial T}=\rho_{\mathrm{p}} s_{\mathrm{p}}^{0}+\rho_{\mathrm{s}} s_{\mathrm{s}}^{0}- \\
\quad-k \frac{\rho_{\mathrm{p}}}{m_{\mathrm{p}}}\left[\log \frac{\rho_{\mathrm{p}}}{\rho_{\mathrm{p}}^{0}}+\frac{1}{2}\left(C_{i j}-\delta_{i j}\right)^{2}\right]
\end{gathered}
$$

where $s^{0}$ is the specific entropy of a pure phase,

$$
\alpha_{i j}=\frac{\partial G}{\partial C_{i j}}=k T \frac{\rho_{\mathrm{p}}}{m_{\mathrm{p}}}\left(C_{i j}-\delta_{i j}\right)
$$

and

$$
P_{i}=\frac{\partial G}{\partial w_{i}}=\frac{\rho_{\mathrm{p}} \rho_{\mathrm{s}}}{\rho} w_{i} \simeq \rho_{\mathrm{p}} w_{i}
$$

with

$$
\mathbf{w}=\mathbf{v}_{\mathbf{p}}-\mathbf{v}_{\mathbf{s}} \text {. }
$$

If we now consider the total energy

$$
U=G+T S-p+\frac{1}{2} \rho V^{2}
$$

we deduce from equation (6) the so-called Gibbs relation

$$
\begin{aligned}
T \mathrm{~d} S=\mathrm{d} U & -\left(\mu_{\mathrm{p}}-\frac{V^{2}}{2}\right) \mathrm{d} \rho_{\mathrm{p}}-\left(\mu_{\mathrm{s}}-\frac{V^{2}}{2}\right) \mathrm{d} \rho_{\mathrm{s}}- \\
& -\alpha_{i j} \mathrm{~d} C_{i j}-P_{i} \mathrm{~d} w_{i}-V_{i} \mathrm{~d}\left(\rho V_{i}\right) .
\end{aligned}
$$

3. The entropy production (first stage). - To describe the evolution in time of the solution we must have an evolution equation for each of the thermo- 
dynamic variables $\rho_{\mathrm{p}}, \rho_{\mathrm{s}}, \rho V_{i}, U, w_{i}$ and $C_{i j}$. Galilean invariance is satisfied with the following equations

$$
\begin{aligned}
& \frac{\partial \rho_{\mathrm{p}}}{\partial t}+\nabla \cdot\left(\rho_{\mathrm{p}} \mathbf{V}+\mathbf{J}\right)=0 \\
& \frac{\partial \rho_{\mathrm{s}}}{\partial t}+\nabla \cdot\left(\rho_{\mathrm{s}} \mathbf{V}-\mathbf{J}\right)=0 \\
& \frac{\partial \rho \mathbf{V}}{\partial t}+\nabla \cdot(\rho \overline{\overline{V V}}+\overline{\bar{\pi}})=\rho \mathbf{g} \quad\left(\pi_{i j}=\pi_{j i}\right) \\
& \frac{\partial U}{\partial t}+\nabla \cdot(U \mathbf{V}+\mathbf{V} \cdot \overline{\bar{\pi}}+\mathbf{Q})=\rho \mathbf{g} \cdot \mathbf{V} \\
& \frac{\partial \mathbf{w}}{\partial t}+(\mathbf{V} \cdot \mathbf{V}) \mathbf{w}=\mathbf{F}-\mathbf{\nabla} \cdot \overline{\bar{T}} \\
& \frac{\partial \overline{\bar{C}}}{\partial t}+(\mathbf{V} \cdot \mathbf{\nabla}) \overline{\bar{C}}=\overline{\bar{R}}
\end{aligned}
$$

Combining these equations with the Gibbs relation(13) we can derive the balance equation for the entropy

$$
\begin{array}{r}
\frac{\partial S}{\partial t}+\nabla .(S \mathbf{V}+\mathbf{S})=-\frac{1}{T}\left[\mathbf{S} . \nabla T+\mathbf{J} . \nabla\left(\mu_{\mathrm{p}}-\mu_{\mathrm{s}}\right)+\mathbf{F} . \mathbf{P}+\right. \\
+(\overline{\bar{\pi}}-p \overline{\bar{I}}): \overline{\overline{\nabla V}}+\overline{\bar{T}}: \overline{\overline{\nabla P}}+\overline{\bar{R}}: \overline{\bar{\alpha}}]
\end{array}
$$

where the entropy flux $\mathbf{S}$ is defined as

$$
T \mathbf{S}=\mathbf{Q}-\left(\mu_{\mathrm{p}}-\mu_{\mathrm{s}}\right) \mathbf{J}-\mathbf{P} . \overline{\bar{T}} .
$$

At this point, the usual procedure is to deduce from the entropy production (the right-hand side of eq. (20)) the expressions for the hitherto unknown fluxes (S, J, F, $\overline{\bar{\pi}}, \overline{\bar{T}}$ and $\overline{\bar{R}}$ ) as functions of the thermodynamic forces $\left(\nabla T, \nabla\left(\mu_{\mathrm{p}}-\mu_{\mathrm{s}}\right), \mathbf{P}, \overline{\overline{\nabla V}}, \overline{\overline{\nabla P}}\right.$ and $\left.\overline{\bar{\alpha}}\right)$. The resulting expressions are very complicated if one looks for completeness. Here we shall adopt a somewhat different approach by referring to the dumbbell model to get a sound expression for the flux $\overline{\bar{R}}$, and by proposing plausible expressions for $\mathbf{J}, \mathbf{S}$ and $\overline{\bar{\pi}}$ which explicitly take into account the presence of two different velocities $\mathbf{V}_{\mathrm{p}}$ and $\mathbf{V}_{\mathrm{s}}$.

4. The basic hypotheses concerning the transport fluxes. - The equation of conservation for the polymer mass is actually

$$
\frac{\partial \rho_{\mathrm{p}}}{\partial t}+\nabla \cdot \rho_{\mathrm{p}} \mathbf{V}_{\mathrm{p}}=0
$$

Comparing with (14) we deduce with the help of (11)

$$
\mathbf{J}=\rho_{\mathrm{p}}\left(\mathbf{V}_{\mathbf{p}}-\mathbf{V}\right) \equiv \mathbf{P} .
$$

Moreover the conservation of momentum must certainly appear as

$$
\frac{\partial \rho \mathbf{V}}{\partial t}+\nabla \cdot\left(\rho_{\mathrm{p}}{\overline{\overline{V_{\mathrm{p}} V}}}_{\mathrm{p}}+\rho_{\mathrm{s}}{\overline{\overline{V_{\mathrm{s}}}}}_{\mathrm{s}}+p \overline{\bar{I}}+\overline{\bar{\pi}}^{*}\right)=\rho \mathbf{g}
$$

hence, comparing with (16) we should write

$$
\overline{\bar{\pi}}=p \overline{\bar{I}}+\overline{\overline{P w}}+\overline{\bar{\pi}}^{*}
$$

where $\overline{\bar{\pi}}^{*}$ is yet undetermined.

Concerning the entropy flux $\mathbf{S}$, things are a bit more delicate. If the specific entropies $s_{\mathrm{p}}^{0}$ and $s_{\mathrm{s}}^{0}$ indeed move with $\mathbf{V}_{\mathrm{p}}$ and $\mathbf{V}_{\mathrm{s}}$ respectively it is generally difficult to decide with which velocity the entropy of mixing and the entropy of deformation are convected. But if we notice that for dilute solutions, both these entropies are proportional to $\rho_{\mathrm{p}}$ (see eq. (9)) it seems reasonable to propose that in this limit they are both convected with $\mathbf{V}_{\mathbf{p}}$. Consequently, defining the effective polymer specific entropy as

$$
s_{\mathrm{p}}=s_{\mathrm{p}}^{0}-\frac{k}{m_{\mathrm{p}}}\left[\log \frac{\rho_{\mathrm{p}}}{\rho_{\mathrm{p}}^{0}}+\frac{1}{2}\left(C_{i j}-\delta_{i j}\right)^{2}\right]
$$

we see that the total entropy flux should be written as the sum

$$
\rho_{\mathrm{p}} s_{\mathrm{p}} \mathbf{V}_{\mathrm{p}}+\rho_{\mathrm{s}} s_{\mathrm{s}}^{0} \mathbf{V}_{\mathrm{s}}+\mathbf{s}=S \mathbf{V}+\left(s_{\mathrm{p}}-s_{\mathrm{s}}^{0}\right) \mathbf{P}+\mathbf{s},
$$

with $S$ defined by equation (9). Now by comparing with the left-hand side of equation (20) we deduce that

$$
\mathbf{S}=\left(s_{\mathrm{p}}-s_{\mathrm{s}}^{0}\right) \mathbf{P}+\mathbf{s} .
$$

Lastly the dumbbell model [18] suggests that for a dilute solution the evolution in time of the polymer shape is

$$
\begin{aligned}
\frac{\partial \overline{\bar{C}}}{\partial t}+\left(\mathbf{V}_{\mathrm{p}} \cdot \mathbf{\nabla}\right) \overline{\bar{C}} & =\overline{\bar{\omega}}_{\mathrm{s}} \cdot \overline{\bar{C}}+\overline{\bar{C}} \cdot \overline{\bar{\omega}}_{\mathrm{s}}+ \\
& +\beta\left(\overline{\bar{D}}_{\mathrm{s}} \cdot \overline{\bar{C}}+\overline{\bar{C}} \cdot \overline{\bar{D}}_{\mathrm{s}}\right)-\frac{1}{\tau}(\overline{\bar{C}}-\overline{\bar{I}})
\end{aligned}
$$

and this implies that our $\overline{\bar{R}}$ in (19) is likely to take the form

$$
\begin{aligned}
\overline{\bar{R}}=-\frac{1}{\rho_{\mathrm{p}}}(\mathbf{P} \cdot \nabla) \overline{\bar{C}}+\overline{\bar{\omega}}_{\mathrm{s}} \cdot \overline{\bar{C}}+\overline{\bar{C}} \cdot \overline{\bar{\omega}}_{\mathrm{s}}+ \\
+\beta\left(\overline{\bar{D}}_{\mathrm{s}} \cdot \overline{\bar{C}}+\overline{\bar{C}} \cdot \overline{\bar{D}}_{\mathrm{s}}\right)+\overline{\bar{r}}
\end{aligned}
$$

where $\overline{\bar{r}}$ is related to the relaxation of the polymer deformation. The above equation (26) means that in the frame moving with the particle velocity and rotating with the angular velocity $\overline{\bar{\omega}}_{\mathrm{s}}$ of the solvent, the polymer deformation is the result of a relaxation towards the equilibrium (spherical) shape and of the action of the solvant deformation rate $\overline{\bar{D}}$ (the symmetric part of the tensor $\overline{\overline{\nabla V}}_{\mathrm{s}}$ ) with an efficiency coefficient $\beta$ that we expect to be in the range of zero (for rigid molecules) to one (for perfectly deformable ones) and which increases when the hydrodynamic interaction between the monomers decreases [19]. 
5. The entropy production (second stage). - Taking into account the following identity

$$
\overline{\overline{\nabla V}}_{\mathrm{s}}=\overline{\overline{\nabla V}}-\overline{\left.\overline{\nabla\left(P / \rho_{\mathrm{s}}\right.}\right)} \simeq \overline{\overline{\nabla V}}-\frac{1}{\rho} \overline{\overline{\nabla P}},
$$

(the last equality is valid for dilute solutions only) we finally transform equation (20) with the help of equations (22), (23), (25) and (27) into

$$
\begin{aligned}
\frac{\partial S}{\partial t}+\nabla \cdot(S \mathbf{V}+\mathbf{S})=-\frac{1}{T}[\mathbf{s} \cdot \nabla T & +\mathbf{f} \cdot \mathbf{P}+\overline{\bar{\tau}}: \overline{\overline{\nabla V}}+ \\
& =\overline{\bar{t}}: \overline{\overline{\nabla P}}+\overline{\bar{r}: \overline{\bar{\alpha}}]}
\end{aligned}
$$

where the final fluxes appearing in the entropy balance are related to the former ones appearing in equations (14) to (19) by the following relations (some of which were already stated)

$\mathbf{J}=\mathbf{P}$

$\mathbf{S}=\left(s_{\mathrm{p}}-s_{\mathrm{s}}^{0}\right) \mathbf{P}+\mathbf{s}$

$\overline{\bar{R}}=-\frac{1}{\rho_{\mathrm{p}}}(\mathbf{P} \cdot \nabla) \overline{\bar{C}}+\overline{\bar{\omega}}_{\mathrm{s}} \cdot \overline{\bar{C}}+\overline{\bar{C}} \cdot \overline{\bar{\omega}}_{\mathrm{s}}+$

$$
+\beta\left(\overline{\bar{C}} \cdot \overline{\bar{D}}_{\mathrm{s}}+\overline{\bar{D}}_{\mathrm{s}} \cdot \overline{\bar{C}}\right)+\overline{\bar{r}}
$$

$\overline{\bar{\pi}}=p \overline{\bar{I}}+\overline{\overline{P w}}-\beta(\overline{\bar{\alpha}} \cdot \overline{\bar{C}}+\overline{\bar{C}} \cdot \overline{\bar{\alpha}})+\overline{\bar{\tau}}$

$\overline{\bar{T}}=\frac{\beta}{\rho}(\overline{\bar{\alpha}} \cdot \overline{\bar{C}}+\overline{\bar{C}} \cdot \overline{\bar{\alpha}})+\overline{\bar{t}}$

$\mathbf{F}=-\left(s_{\mathrm{p}}-s_{\mathrm{s}}^{0}\right) \nabla T-\nabla\left(\mu_{\mathrm{p}}-\mu_{\mathrm{s}}\right)+\frac{1}{\rho_{\mathrm{p}}} \alpha_{i j} \nabla C_{i j}-$ $-(\mathbf{w} \cdot \boldsymbol{\nabla}) \mathbf{V}+\mathbf{f}$,

while the energy flux is given by

$$
\mathbf{Q}=T \mathbf{S}+\left(\mu_{\mathrm{p}}-\mu_{\mathrm{s}}\right) \mathbf{J}+\mathbf{P} \cdot \overline{\bar{T}} \text {. }
$$

The main result of this section is represented by the expressions (30) and (31) for $\overline{\bar{T}}$ and $\mathbf{F}\left({ }^{1}\right)$. We have just shown how these terms entering the relative velocity equation of motion (18) may be obtained unambiguously provided the relatice velocity dependence of the fluxes $\mathbf{S}, \mathbf{J}, \overline{\bar{\pi}}$ and $\overline{\bar{R}}$ is known or at least guessed at. It must be stressed that other choices are possible. One may claim for instance that the entropy of mixing and the entropy of deformation are convected with the centre of mass velocity $\mathbf{V}$, and also that the polymer shape depends on $\overline{\bar{\omega}}$ and $\overline{\bar{D}}$ (the components of $\overline{\overline{\nabla V}}$ ) instead of $\overline{\bar{\omega}}_{\mathrm{s}}$ and $\overline{\bar{D}}_{\mathrm{s}}$. In this case we would have found with the same procedure

$$
\begin{aligned}
& \frac{\partial S}{\partial t}+\nabla \cdot(S \mathbf{V}+\mathbf{S})=-\frac{1}{T}\left[\mathbf{s}^{*} \cdot \nabla T+\mathbf{f}^{*} \cdot \mathbf{P}+\overline{\bar{\tau}} *: \overline{\overline{\nabla V}}+\right.
\end{aligned}
$$

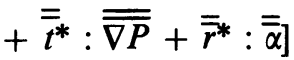

( $\left.{ }^{1}\right)$ The expression (29) for $\overline{\bar{\pi}}$ is also of utmost importance but its implications were already analysed in [20]. with

$$
\begin{aligned}
& \mathbf{S}=\left(s_{\mathrm{p}}^{0}-s_{\mathrm{s}}^{0}\right) \mathbf{P}+\mathbf{s}^{*} \\
& \overline{\bar{R}}=\overline{\bar{\omega}} \overline{\bar{C}}+\overline{\bar{C}} \cdot \overline{\bar{\omega}}+\beta(\overline{\bar{C}} \cdot \overline{\bar{D}}+\overline{\bar{D}} \cdot \overline{\bar{C}})+\overline{\bar{r}}^{*} \\
& \overline{\bar{\pi}}=p \overline{\bar{I}}+\overline{\overline{P w}}-\beta\left(\overline{\bar{\alpha}} \cdot \overline{\bar{C}}+\overline{\bar{C} \cdot \overline{\bar{\alpha}})+\overline{\bar{\tau}}^{*}}\right. \\
& \overline{\bar{T}}=\overline{\overline{t^{*}}} \\
& \mathbf{F}=-\left(s_{\mathrm{p}}^{0}-s_{\mathrm{s}}^{0}\right) \nabla T-\nabla\left(\mu_{\mathrm{p}}-\mu_{\mathrm{s}}\right)- \\
& \quad-(\mathbf{w} \cdot \nabla) \mathbf{V}+\mathbf{f}^{*} .
\end{aligned}
$$

We shall later see that the polymer migration predicted by the expressions for $\overline{\bar{T}}$ and $\mathbf{F}$ is very different for the couple (30) and (31) as compared to (33) and (34) so that we have experimental means to determine the right proposal. Moreover we shall also compare the above thermodynamic results with those obtained from a kinetic theory approach. But before doing this we must first express in a more conventional way the relative velocity equation of motion.

6. The equation of motion for the relative velocity. Our calculation will be made in three steps :

i) First, we use the thermodynamic identity

$$
\nabla\left(\mu_{\mathrm{p}}^{0}-\mu_{\mathrm{s}}^{0}\right)+\left(s_{\mathrm{p}}^{0}-s_{\mathrm{s}}^{0}\right) \nabla T=\left(\frac{1}{\rho_{\mathrm{p}}^{0}}-\frac{1}{\rho_{\mathrm{s}}^{0}}\right) \nabla p
$$

which is a consequence of the Gibbs-Duhem relation for two phases with equal pressures and temperatures. For non-buoyant mixtures $\left(\rho_{\mathrm{p}}^{0}=\rho_{\mathrm{s}}^{0}\right)$ the right-hand side of the above equation is null.

ii) The kinetic part of the chemical potential difference (see Eqs. (7) and (8)) can be transformed as follows

$$
\begin{aligned}
& \frac{1}{2} \nabla\left(\mathbf{V}_{\mathrm{p}}-\mathbf{V}\right)^{2}-\frac{1}{2} \nabla\left(\mathbf{V}_{\mathrm{s}}-\mathbf{V}\right)^{2}= \\
& \quad=\left(\mathbf{V}_{\mathrm{p}} \cdot \nabla\right) \mathbf{V}_{\mathrm{p}}-\left(\mathbf{V}_{\mathrm{s}} \cdot \boldsymbol{\nabla}\right) \mathbf{V}_{\mathrm{s}}-(\mathbf{V} \cdot \nabla) \mathbf{w}-(\mathbf{w} \cdot \nabla) \mathbf{V}+ \\
& +\left(\mathbf{V}_{\mathrm{p}}-\mathbf{V}\right) \times\left[\nabla \times\left(\mathbf{V}_{\mathrm{p}}-\mathbf{V}\right)\right]-\left(\mathbf{V}_{\mathrm{s}}-\mathbf{V}\right) \times\left[\nabla \times\left(\mathbf{V}_{\mathrm{s}}-\mathbf{V}\right)\right] .
\end{aligned}
$$

Because of their orthogonality to the relative velocity $\mathbf{w}$, the underlined terms are irrelevant as far as the entropy production is concerned. This means that $\mathbf{F}$ in equations (31) or (34) is defined up to an arbitrary term orthogonal to w. Whether such a «gyroscopic» term actually exists or not is still obscure in the author's mind.

iii) Lastly, one can verify with equations (7), (10) and (24) that in the case of dilute solutions

$\nabla\left(\Delta \mu_{\mathrm{p}}\right)=\frac{1}{\rho_{\mathrm{p}}} \alpha_{i j} \nabla C_{i j}-\left(s_{\mathrm{p}}-s_{\mathrm{p}}^{0}\right) \nabla T+\frac{k T}{m_{\mathrm{p}}} \frac{\nabla \rho_{\mathrm{p}}}{\rho_{\mathrm{p}}}$

where

$$
\Delta \mu_{\mathrm{p}}=\mu_{\mathrm{p}}-\mu_{\mathrm{p}}^{0}-\frac{1}{2}\left(\mathbf{V}_{\mathrm{p}}-\mathbf{V}\right)^{2} .
$$

With the above three results and the definition

$$
\frac{\mathrm{D}_{k} \mathbf{V}_{k}}{\mathrm{D} t}=\frac{\partial \mathbf{V}_{k}}{\partial t}+\left(\mathbf{V}_{k} \cdot \nabla\right) \mathbf{V}_{k}
$$


equation (18) can be transformed into

$$
\begin{aligned}
& \frac{\mathrm{D}_{\mathrm{p}} \mathbf{V}_{\mathrm{p}}}{\mathrm{D} t}-\frac{\mathrm{D}_{\mathrm{s}} \mathbf{V}_{\mathrm{s}}}{\mathrm{D} t}+\left(\frac{1}{\rho_{\mathrm{p}}^{0}}-\frac{1}{\rho_{\mathrm{s}}^{0}}\right) \nabla p= \\
& \quad=-\frac{k T}{m_{\mathrm{p}}} \frac{\nabla \rho_{\mathrm{p}}}{\rho_{\mathrm{p}}}-\nabla \cdot\left[\frac{\beta}{\rho}(\overline{\bar{\alpha}} \cdot \overline{\bar{C}}+\overline{\bar{C}} \cdot \overline{\bar{\alpha}})\right]+\mathbf{f}-\nabla \cdot \overline{\bar{t}}(36)
\end{aligned}
$$

with the hypothesis leading to equations (30) and (31), or into

$$
\begin{aligned}
\frac{\mathrm{D}_{\mathrm{p}} \mathbf{V}_{\mathrm{p}}}{\mathrm{D} t}-\frac{\mathrm{D}_{\mathrm{s}} \mathbf{V}_{\mathrm{s}}}{\mathrm{D} t}+\left(\frac{1}{\rho_{\mathrm{p}}^{0}}-\frac{1}{\rho_{\mathrm{s}}^{0}}\right) \nabla p= \\
=-\nabla\left(\Delta \mu_{\mathrm{p}}\right)+\mathbf{f}^{*}-\nabla \cdot \overline{\bar{t}^{*}}
\end{aligned}
$$

with the hypothesis leading to equations (33) and (34) (the expression for $\nabla\left(\Delta \mu_{\mathrm{p}}\right)$ is given by Eq. (35)).

Expressions (36) and (37) are the central results of this paper and we end this section with the expressions for $\mathbf{f}\left(\right.$ or $\mathbf{f}^{*}$ )and $\overline{\bar{t}}$ (or $\overline{\bar{t}^{*}}$ ). From either of the entropy production equations (28) or (32), we deduce that

$$
\mathbf{f}=-\frac{v_{1}}{\rho_{\mathrm{p}}^{0} r_{0}^{2}} \mathbf{P}
$$

and

$$
\overline{\bar{t}}=-\frac{v_{2}}{\rho_{\mathrm{p}}^{0}} \overline{\overline{\nabla P}}-v_{3} \overline{\overline{\nabla V}}
$$

where the $v_{k}$ are kinematic viscosities with $v_{1}$ and $v_{2}$ positive scalars while the sign of $v_{3}$ is arbitrary (the other terms like $\rho_{\mathrm{p}}^{0}$ and $r_{0}$ are introduced for dimensional convenience only). For a dilute solution

$$
\mathbf{V} \simeq \mathbf{V}_{\mathrm{s}} \quad \text { and } \quad \mathbf{P} \simeq \rho\left(\mathbf{V}-\mathbf{V}_{\mathrm{s}}\right)
$$

and in this approximation

$$
\mathbf{f}-\nabla \cdot \overline{\bar{t}}=-\frac{v_{1}}{\rho_{\mathrm{p}}^{0} r_{0}^{2}} \mathbf{P}+v_{3} \nabla^{2} \mathbf{V}_{\mathrm{s}}
$$

This result must be compared with the viscous force $\mathbf{F}_{\mathrm{v}}$ acting on a particle in an arbitrary velocity field, the so-called Faxen's force [12]

$$
\mathbf{F}_{\mathrm{v}}=-\xi\left(\mathbf{V}_{\mathrm{p}}-\mathbf{V}_{\mathrm{s}}-\frac{r_{0}^{2}}{6} \nabla^{2} \mathbf{V}_{\mathrm{s}}\right)
$$

where

$$
\xi=6 \pi \eta_{\mathrm{s}} r_{0}
$$

is the friction coefficient and $\eta_{\mathrm{s}}$ the solvent viscosity. It is not difficult to convince oneself that for a dilute solution

$$
\mathbf{f}-\nabla \cdot \overline{\bar{t}}=\mathrm{F}_{\mathrm{v}} / m_{\mathrm{p}}=\mathbf{f}^{*}-\nabla \cdot \overline{\bar{t}} *
$$

where $m_{\mathrm{p}}$ is the polymer mass and consequently

$$
v_{1}=\frac{1}{\rho_{\mathrm{p}}} \frac{\xi \rho_{\mathrm{p}}^{0} r_{0}^{2}}{m_{\mathrm{p}}} \simeq \frac{9}{2} \frac{\eta_{\mathrm{s}}}{\rho_{\mathrm{p}}}
$$

and

$$
v_{3}=\frac{3}{4} \frac{\eta_{\mathrm{s}}}{\rho}
$$

We shall not bother further with the exact values of $v_{1}$ and $v_{3}$ and, instead, we shall consider henceforth that in both equations (36) and (37), the viscous force

$$
\mathbf{f}-\nabla \cdot \overline{\bar{t}} \text { or } \mathbf{f}^{*}-\nabla \cdot \overline{\overline{t^{*}}}
$$

is given by equation (40) where $F_{v}$ is the Faxen's force (38).

7. Comparison with previous work. - 7.1 THE ENTROPIC FORCE SCHEME. - If we consider a neutrallybuoyant mixture $\left(\rho_{\mathrm{p}}^{0}=\rho_{\mathrm{s}}^{0}\right)$ in a stationary motion with negligible acceleration

$$
\frac{\mathrm{D}_{\mathrm{p}} \mathbf{V}_{\mathrm{p}}}{\mathrm{D} t}=\frac{\mathrm{D}_{\mathrm{s}} \mathbf{V}_{\mathrm{s}}}{\mathrm{D} t} \simeq 0
$$

then from equations (37) and (40) we get

$$
\mathbf{F}_{\mathrm{v}} / m_{\mathrm{p}}=\nabla\left(\Delta \mu_{\mathrm{p}}\right)
$$

with $\nabla\left(\Delta \mu_{\mathrm{p}}\right)$ and $\mathrm{F}_{\mathrm{v}}$ respectively given by equations (35) and (38). The resulting expression for the diffusion mass flux $\mathbf{J}$ is thus

$$
\begin{aligned}
\mathbf{J}=\rho_{\mathrm{p}}\left(\mathbf{V}_{\mathrm{p}}-\mathbf{V}_{\mathrm{s}}\right) & \\
=\rho_{\mathrm{p}} \frac{r_{0}^{2}}{6} \nabla^{2} \mathbf{V}_{\mathrm{s}} & -\frac{k T}{\xi}\left[\nabla \rho_{\mathrm{p}}+\frac{m_{\mathrm{p}}}{k T} \alpha_{i j} \nabla C_{i j}-\right. \\
& \left.\quad-\frac{\rho_{\mathrm{p}} m_{\mathrm{p}}}{k T}\left(s_{\mathrm{p}}-s_{\mathrm{p}}^{0}\right) \nabla T\right]
\end{aligned}
$$

where $\alpha_{i j}$ is given by (10). Except for the terms involving $\nabla^{2} V_{\mathrm{s}}$ and $\nabla T$, the above expression is exactly the one found by Tirrell and Malone [14]. The name entropic force comes from the shape dependence of the chemical potential which is mainly entropic in origin.

7.2 THE SAJ FORCE SCHEME. - Again for neutrallybuoyant mixtures and negligibly accelerated flows, equations (36) with (40) merges into

$$
\mathbf{F}_{\mathrm{v}} / m_{\mathrm{p}}=\frac{k T}{m_{\mathrm{p}}} \frac{\nabla \rho_{\mathrm{p}}}{\rho_{\mathrm{p}}}+\nabla \cdot\left[\frac{\beta}{\rho_{\mathrm{p}}^{0}}(\overline{\bar{\alpha}} \cdot \overline{\bar{C}}+\overline{\bar{C}} \cdot \overline{\bar{\alpha}})\right]
$$

and the diffusion mass flux is in this case

$$
\begin{aligned}
& \mathbf{J}=\rho_{\mathrm{p}} \frac{r_{0}^{2}}{6} \nabla^{2} \mathbf{V}_{\mathrm{s}}- \\
& -\frac{k T}{\xi}\left\{\nabla \rho_{\mathrm{p}}+\frac{m_{\mathrm{p}} \rho_{\mathrm{p}}}{k T} \nabla \cdot\left[\frac{\beta}{\rho_{\mathrm{p}}^{0}}(\overline{\bar{\alpha}} \cdot \overline{\bar{C}}+\overline{\bar{C}} \cdot \overline{\bar{\alpha}})\right]\right\} .
\end{aligned}
$$

Note the quite different structure between (42) and (44), in particular the presence of $\overline{\bar{\alpha}}$ inside a divergence term in (44). As a consequence, expression (44) is likely to include anisotropic terms involving $\nabla \rho_{\mathrm{p}}$ 
and $\nabla T$ (due to the $\rho_{\mathrm{p}}$ and $T$ dependence of $\overline{\bar{\alpha}}$, see eq. (10)) while such a possibility is excluded from (42). Moreover the part of (42) depending on the polymer shape $\overline{\bar{C}}$ only implies a cross-streamlines migration (see ref. [14]) while the more complicated structure of the corresponding part of (44) is compatible with cross-streamlines as well as along-streamlines migration, as we shall later see. The structure of expression (44) thus bears many resemblances to that deduced by Sekhon, Armstrong and Jones from a kinetic theory approach [13] but the physical interpretation of this part of the mass flux depending on the polymer deformation needs some clarification.

8. Re-interpretation of the SAJ-like force. - Let us consider the relative position vector $\mathbf{r}$ separating the centres of two spheres submitted to a non-uniform velocity field with a rotation vector $\vec{\omega}_{\mathrm{s}}$ and a gradient of strain rate $\overline{\bar{D}}_{\mathrm{s}}$. If the two spheres interact via a force $\mathbf{F}$ (including the Brownian motion force) we can write with Batchelor [21, 22]

$$
\frac{\mathrm{d} \mathbf{r}}{\mathrm{d} t}=\vec{\omega}_{\mathrm{s}} \times \mathbf{r}+\overline{\bar{D}}_{\mathrm{s}} \cdot \mathbf{r}-\left\{A(r) \frac{\overline{\overline{r r}}}{r^{2}}+B(r)\left(\overline{\bar{I}}-\frac{\overline{\overline{r r}}}{r^{2}}\right)\right\} \cdot \overline{\bar{D}}_{\mathrm{s}} \cdot \mathbf{r}+\frac{1}{\xi}\left\{G(r) \frac{\overline{\overline{r r}}}{r^{2}}+H(r)\left(\overline{\bar{I}}-\frac{\overline{\overline{r r}}}{r^{2}}\right)\right\} \mathbf{F}
$$

In the dumbbell model, the polymer molecule is compared to such a system of two interacting spheres and $\mathbf{r}$ now represents the deformation of the molecule. In their dumbbell model approach to the diffusion flux, Sekhon, Armstrong and Jones [13] emphasized on the role of the hydrodynamic interactions in the anisotropic nature of the diffusion tensor, i.e. on the terms $G$ and $H$ of the above equation. But hydrodynamic interactions have another role which is the modification of the effective velocity field seen by each monomer due to the presence of other monomers and this is represented by the terms $A$ and $B$ of the above equation. When statistically averaging equation (45) to get an equation like (26) for

$$
C_{i j}=3\left\langle r_{i} r_{j}\right\rangle / r_{0}^{2},
$$

the role of the anisotropic diffusion tensor is merely to renormalize the relaxation time for the polymer deformation [18], while the role of $A(r)$ and $B(r)$ is to appear as the coefficient $\beta$ in equation (26), this coefficient playing a crucial role in the expression (44) of the diffusion flux. Thus, if the SAJ force had indeed something to do with hydrodynamic interactions inside a polymer molecule, we have the feeling that the modification of the effective velocity field is far more important than the anisotropy of the diffusion tensor.

9. Migration in a Poiseuille flow. - Let us consider a Poiseuille flow with the simple velocity field $V_{z}(r)$ for the solvent. The shape of the polymer molecule is given by the solution of equation (26) which is

$$
\begin{aligned}
& C_{r r}=\left[1+(1-\beta) X^{2}\right]\left[1+\left(1-\beta^{2}\right) X^{2}\right]^{-1} \\
& C_{z z}=\left[1+(1+\beta) X^{2}\right]\left[1+\left(1-\beta^{2}\right) X^{2}\right]^{-1} \\
& C_{r z}=\beta X\left[1+\left(1-\beta^{2}\right) X^{2}\right]^{-1} \\
& C_{r \theta}=C_{z \theta}=0, \quad C_{\theta \theta}=1
\end{aligned}
$$

where $X(r)$ is the Deborah number

$$
X=\tau \frac{\mathrm{d} V_{z}}{\mathrm{~d} r}
$$

while $\tau$ is the Zimm relaxation time. Note that, except for the case $\beta=1$ corresponding to the absence of hydrodynamic interactions, the polymer shape is always finite whatever the Deborah number is. With $\overline{\bar{\alpha}}$ given by equation (10), we are then able to express the diffusion mass flux as a function of $V_{z}$ and its spatial derivatives, using either equation (42) or equation (44). If we are confident that equation (42) is the right answer we get for isothermal Poiseuille flow

$$
\begin{aligned}
& J_{z}=\rho_{\mathrm{p}} \frac{r_{0}^{2}}{6} \frac{1}{r} \frac{\mathrm{d}}{\mathrm{d} r}\left(r \frac{\mathrm{d} V_{z}}{\mathrm{~d} r}\right) \\
& J_{r}=-\frac{k T}{\xi}\left[\frac{\mathrm{d} \rho_{\mathrm{p}}}{\mathrm{d} \dot{r}}+\rho_{\mathrm{p}}\left(C_{r r}-1\right) \frac{\mathrm{d} C_{r r}}{\mathrm{~d} r}+\rho_{\mathrm{p}}\left(C_{z z}-1\right) \frac{\mathrm{d} C_{z z}}{\mathrm{~d} r}+\rho_{\mathrm{p}} C_{r z} \frac{\mathrm{d} C_{r z}}{\mathrm{~d} r}\right] \\
& J_{\theta}=0
\end{aligned}
$$

but if we prefer equation (44) we get again for an isothermal flow with $\beta$ and $\rho_{\mathrm{p}}^{0}$ taken as constants,

$$
\begin{aligned}
& J_{z}=\rho_{\mathrm{p}} \frac{r_{0}^{2}}{6} \frac{1}{r} \frac{\mathrm{d}}{\mathrm{d} r}\left(r \frac{\mathrm{d} V_{z}}{\mathrm{~d} r}\right)-\frac{k T}{\xi} \frac{2 \beta \rho_{\mathrm{p}}}{\rho_{\mathrm{p}}^{0}} \frac{1}{r} \frac{\mathrm{d}}{\mathrm{d} r}\left[r \rho_{\mathrm{p}} C_{r z}\left(C_{r r}+C_{z z}-1\right)\right] \\
& J_{r}=-\frac{k T}{\xi}\left\{\frac{\mathrm{d} \rho_{\mathrm{p}}}{\mathrm{d} r}+\frac{2 \beta \rho_{\mathrm{p}}}{\rho_{\mathrm{p}}^{0}} \frac{1}{r} \frac{\mathrm{d}}{\mathrm{d} r}\left[r \rho_{\mathrm{p}}\left(C_{r r}^{2}+C_{r z}^{2}-C_{r r}\right)\right]\right\} \\
& J_{\theta}=0 .
\end{aligned}
$$


If we express the components $C_{i j}$ as a function of the Deborah number, the resulting expressions are very complicated. For this reason we limit ourselves to the low Deborah number limit $(X \ll 1)$ and the cross-streamlines component of $\mathbf{J}$ is in this case

$$
J_{r}=-\frac{k T}{\xi}\left[\frac{\mathrm{d} \rho_{\mathrm{p}}}{\mathrm{d} r}+\rho_{\mathrm{p}} \beta^{2} \tau^{2} \frac{\mathrm{d} V_{z}}{\mathrm{~d} r} \frac{\mathrm{d}^{2} V_{z}}{\mathrm{~d} r^{2}}\right]
$$

or

$$
J_{r}=-\frac{k T}{\bar{\xi}}\left\{\frac{\mathrm{d} \rho_{\mathrm{p}}}{\mathrm{d} r}+\frac{2 \beta \rho_{\mathrm{p}}}{\rho_{\mathrm{p}}^{0}} \beta(2 \beta-1) \tau^{2} \frac{1}{r} \frac{\mathrm{d}}{\mathrm{d} r}\left[r \rho_{\mathrm{p}}\left(\frac{\mathrm{d} V_{z}}{\mathrm{~d} r}\right)^{2}\right]\right\}
$$

depending on which expression for $\mathbf{J}$ we take for granted. Anyway the above expressions confirm the existence of an anisotropic diffusion in the SAJ force scheme (expressed by the presence of $\mathrm{d} \rho_{\mathrm{p}} / \mathrm{d} r$ in $J_{z}$ ), and a much weaker effect (of the order $2 \beta \rho_{\mathrm{p}} / \rho_{\mathrm{p}}^{0}$ ) of the cross-streamlines diffusion with the SAJ force (eq. (50)) as compared to the entropic force (eq. (49)).

10. The Soret effect. - The diffusion mass flux depends on the temperature gradient. This is clearly seen in (42) and the Soret coefficient is in this case the sum of two terms, one of them depending on the polymer deformation as is apparent from inspection of equation (24). For the case of the SAJ force and the corresponding diffusion flux (44), the temperature gradient is implicitly present through the temperature dependence of $\overline{\bar{\alpha}}$ (see eq. (10)) and the remarkable fact is that the Soret coefficient is now proportional to the particle deformation and is thus likely to be unobservable at low Deborah numbers.

11. Conclusion. - We have shown that the wellknown entropic force or the more recent SAJ force can both be justified depending on the hypothesis made concerning the entropy transport and the dynamics of the polymer deformation. If the deformation entropy of the polymer molecules and the entropy of mixing are both convected with the centre of mass velocity $\mathbf{V}$, and if the polymer shape is influenced by the gradient of $\mathbf{V}$, then our thermodynamic analysis shows that for non-accelerated flow of a neutrally-buoyant mixture, the mass flux is given by the gradient of a chemical potential, as in the case for the Fick's law. But if we adopt the (more plausible) hypothesis that both extra entropies are convected with the polymer velocity and that the polymer shape is mainly dependent on the gradient of the solvent velocity, then we get for the diffusion mass flux an expression which is rather similar to that found through a kinetic theory approach by SAJ [13]. Moreover it happens that the Soret coefficient crucially depends on the molecular shape for this last case while only a part of this coefficient is shape dependent in the former case. Lastly the part of the diffusion mass flux depending on the polymer shape is less by a factor $\beta \rho_{\mathrm{p}} / \rho_{\mathrm{p}}^{0}$ (i.e. $\beta$ times the polymer volumic fraction) in the case of the SAJ force compared to the case of the entropic force. Thus the expected effects on migration are generally weaker with the SAJ force.

\section{References}

[1] Maerker, J. M., J. Pet. Technol. 25 (1973) 1307.

[2] Chauveteau, G. and Kohler, N., S.P.E. Meeting, paper no 4745, Tulsa (1974).

[3] Aubert, J. H. and Tirrell, H., Rheol. Acta 19 (1980) 459.

[4] Astarita, G., Marrucci, G. and Palumbo, G., Ind. Eng. Chem. Fundam. 3 (1964) 333.

[5] Kosicki, W., Pasari, S. N., Rao, A. R. K. and Tiu, C., Chem. Eng. Sci. 25 (1970) 41.

[6] Metzner, A. B., Cohen, Y. and Rangel-Nafaile, G., J. Non-Newt. Fluid Mech. 5 (1979) 449.

[7] Busse, W. F., J. Polym. Sci. Part A 2 (1967) 1261.

[8] Schreiber, H. P. and Storey, S. H., J. Polym. Sci. B (1965) 723.

[9] SChaEFER, R. H., LAIKen, N. and Zimm, B. H., Biophys. chem. 2(1974) 180.

[10] BiRD, R. B., J. Non-Newt. Fluid Mech. 5 (1979) 1.

[11] Aubert, J. H. and Tirrell, M., J. Chem. Phys. 72 (1980) 2694 ;

Aubert, J. H., Prager, S. and Tirrell, M., J. Chem. Phys. 73 (1980) 4103.
[12] Brenner, H., Chem. Eng. Sci. 19 (1964) 703.

[13] Sekhon, G., Armstrong, R. C. and Myung S. Jhon, J. Polym. Sci., Phys. (1982).

[14] Tirrell, M. and Malone, M. F., J. Polym. Sci. 15 (1977) 1569.

[15] See for instance G. Weill, Bull. Union Phys. 639 (1981) 293.

[16] Flory, P. J., Principles of Polymer Chemistry (Cornell Univ. Press, Ithaca N.Y.) 1953.

[17] Lhuillier, D., Phys. Fluids 22 (1979) 2033.

[18] Bird, R. B., Hassager, O., Armstrong, R. C. and Curtiss, C. F., Dynamics of Polymeric Liquids : vol. II, Kinetic Theory (Wiley, New York) 1977, ch. 10.

[19] Hinch, E. J., Phys. Fluids 20 (1977) 22.

[20] Lhuillier, D. and Ouibrahim, A., J. Méc. 19 (1980) 725.

[21] Batchelor, G. K. and GreEn, J. T., J. Fluid Mech. 56 (1972) 375.

[22] BATChelor, G. K., J. Fluid Mech. 74 (1976) 1. 